\title{
Early exercise testing and coronary angiography after uncomplicated myocardial infarction
}

\author{
FAWAZ AKHRAS, JAMES UPWARD, ROBIN STOTT, GRAHAM JACKSON
}

\begin{abstract}
In a prospective study 61 patients aged 55 years or less with uncomplicated myocardial infarction underwent treadmill stress testing at two weeks and coronary angiography at six weeks after infarction. Of the 44 patients who had a positive stress test, 43 had additional severe coronary artery disease confirmed by coronary angiography. Of the 17 patients who had a negative stress test for additional disease, coronary angiography identified only single-vessel disease in the infarct area in 15 . The sensitivity of the stress test was $95 \%$ and the specificity $94 \%$, though the number of patients in the study was small. Thus, exercise testing has considerable potential for the early identification of multiple-vessel disease in patients with uncomplicated myocardial infarction.
\end{abstract}

\section{Introduction}

Although exercise-induced depression of the ST segment is known to occur after acute myocardial infarction, there is little information about its importance and usefulness in predicting the extent of additional coronary disease. The feasibility and safety of treadmill exercise testing has been established in patients before discharge from hospital after an uncomplicated myocardial infarction. ${ }^{12}$ Furthermore, pronounced depression of the ST segment during these tests has been shown to predict sudden cardiac death within one year. ${ }^{3}$ On the assumption that the coronary anatomy dictates the prognosis this study was designed to assess the potential value of early exercise testing as a non-invasive means of identifying patients at risk because of additional severe coronary artery disease.

\section{Patients and method}

All patients aged 55 years or less (six women and 55 men) with an uncomplicated acute myocardial infarction were investigated. All patients fulfilled the conventional criteria for acute myocardial infarction with a rise in diagnostic enzyme activities and electrocardiographic changes. Thirty-three patients had had anterior and 28 inferior infarctions. Patients with valvular heart disease or cardiac failure were excluded, as were those being treated with digitalis or beta-blockers and those with left bundle-branch block. No patient took any cardiac drug for at least 48 hours before the exercise testing. All patients underwent both 12-lead maximal exercise testing and coronary angiography.

\section{EXERCISE TESTING}

Patients underwent 12-lead maximal treadmill stress testing two-three weeks after infarction according to the Bruce protocol.

Department of Cardiology, King's College Hospital, London SE5 9RS, and Lewisham Hospital, London SE13 6LH

FAWAZ AKHRAS, MD, research registrar

JAMES UPWARD, MRCP, research registrar

ROBIN STOTT, MRCP, consultant physician

GRAHAM JACKSON, MRCP, consultant cardiologist
Electrocardiograms were recorded before exercise, at completion of each three-minute stage, at peak rate, immediately after exercise, and at one-minute intervals during recovery for three minutes then at two-minute intervals until the recording returned to its resting state. The test was stopped if there was cardiac pain, a notable fall in systolic blood pressure, pronounced depression of the ST segment, dyspnoea, or exhaustion. Horizontal or downsloping depression of the ST segment of $1.5 \mathrm{~mm}$ or more persisting for $80 \mathrm{~ms}$ beyond the $\mathrm{J}$ point was considered to be abnormal. In the presence of resting $\mathrm{T}$-wave changes, with or without $\mathrm{Q}$ waves, the $S T$-segment changes were considered abnormal if further depression of $1 \mathrm{~mm}$ or more from the control recordings occurred. Elevation of the ST segment in the infarct area at rest and during exercise indicates left ventricular dyskinesia $^{4}$ and was not considered to reflect additional ischaemia in the non-infarct area for the purpose of this study. The electrocardiograms were made with a fully automated programmable threechannel electrocardiograph (Marquette Instrument Company, Model 1800 Treadmill, EC Mac 1) complying with American Heart Association standards. Routine blood pressure measurements were taken before, during, and after exercise to monitor left ventricular performance. The exercise test was interpreted before the angiography was performed and, therefore, without any knowledge of the angiographic findings. The following criteria were used to predict further disease on the exercise electrocardiogram: (a) in the presence of an anterior infarct additional ischaemic changes in the inferior and lateral leads would suggest right and circumflex coronary artery disease respectively; (b) in the presence of an inferior infarct additional ischaemic changes in the anterior and lateral leads would indicate left anterior descending and circumflex artery disease respectively.

\section{CORONARY ANGIOGRAPHY}

Cineangiograms were recorded on $35-\mathrm{mm}$ film in multiple views. Stenosis of a major artery was considered to be severe if the diameter was reduced by more than $70 \%$. Left ventriculography was performed in the right anterior oblique projection. In view of the findings of the European Coronary Surgery Study Group at the time of this study coronary angiography was routinely performed for all our patients under 55 years of age to define the coronary anatomy.

\section{Results}

According to the criteria of changes in the ST segment alone, 43 patients had a positive stress test indicating additional severe coronary artery disease. The results of 12-lead maximal exercise testing with coronary angiography are compared in the table. All tests were completed without complications.

Anterior myocardial infarction-Of the 33 patients with anterior infarction, $21(63.6 \%)$ had a positive stress test, which, in all 21 patients, predicted correctly the territory of the additional coronary artery disease. Eleven $(33.3 \%$ ) patients had negative stress tests for additional coronary artery disease, and subsequently their coronary arteriograms showed single-vessel disease to the infarct area. Only

Correlation between 12-lead maximal treadmill testing and coronary angiography soon after infarction in 61 patients ( $n=$ No of patients)

\begin{tabular}{llccc}
\hline & & \multicolumn{3}{c}{ Treadmill test results } \\
\cline { 3 - 5 } $\begin{array}{c}\text { Site of } \\
\text { infarction }\end{array}$ & $\begin{array}{c}\text { Coronary artery disease } \\
\text { by angiography }\end{array}$ & Positive & Negative & Total \\
\hline Anterior $(n=33)$ & Single-vessel disease & 0 & 11 & 11 \\
Inferior $(n=28)$ & Multiple-vessel disease & 21 & 1 & 22 \\
& Single-vessel disease & 1 & 4 & 5 \\
& Multiple-vessel disease & 22 & 1 & 23 \\
\hline
\end{tabular}


one patient had a false-negative test for additional coronary artery disease. The predictive value of a positive test (the percentage of patients with a positive test who have additional disease) was $100 \%$, there being no false-positive results. Thus, the sensitivity of the stress test in patients with anterior infarction was $96 \%$ and the specificity $100 \%$.

Inferior myocardial infarction-Twenty-two $(78.6 \%)$ of the 28 patients with inferior infarction had pronounced depression of the ST segment on treadmill testing, indicating additional coronary artery disease, which was later confirmed by coronary arteriography. Five patients had no additional coronary artery disease and four of these had negative stress tests. Thus, one patient had a false-positive response for additional coronary artery disease. When all patients are considered, two had false-negative and one a false-positive test for additional disease. In 58 of our 61 patients the exercise electrocardiogram correctly identified the coronary anatomy with a predictive value of $98 \%$.

A positive stress test had a sensitivity of $96 \%$, and the specificity of a negative treadmill test soon after infarction was $94 \%$ for the whole group. In all 15 patients who had true negative stress tests for additional ischaemia the exercise electrocardiogram correctly predicted the coronary anatomy.

\section{Discussion}

The development of a non-invasive technique that accurately diagnoses the presence or absence of additional severe coronary artery disease in patients soon after infarction is essential if patients at risk are to be identified and invasive and expensive investigations avoided.

Maximal exercise testing using the standard 12-lead electrocardiograph has proved both highly specific and sensitive for coronary artery disease in our patients. A negative test is less specific in patients with recent inferior infarction; this is probably because of the variable anatomical supply to the inferolateral segment of the heart. Our data indicate that patients with a recent uncomplicated myocardial infarction may have exercise-induced depression of the ST segment indicating ischaemia remote from the infarcted wall. This observation agrees with that of other workers, ${ }^{34}$ who have reported the results of stress testing in patients with previous myocardial damage. ${ }^{5}$

In patients with angina and pronounced three-vessel or left main artery disease surgery offers a better prognosis. ${ }^{6} \mathrm{We}$ have now established the accuracy of early exercise testing after infarction in predicting the extent and severity of additional coronary artery disease. We consider it to be reasonable to apply the same surgical criteria to patients after infarction as to those with angina, since we are concerned with a similar anatomical problem. We believe early exercise testing after infarction is a safe and valuable procedure which should become an essential part of the evaluation of the patient with a so-called uncomplicated myocardial infarction. This is of particular relevance to physicians in peripheral district hospitals who may have easy access to exercise testing facilities but no readily available angiographic services. We decided to determine the usefulness of exercise testing in this context with a view to reducing the need for unnecessary coronary angiography in these patients. In our hands, with careful supervision of the 12-lead electrocardiogram and systolic blood pressure, maximal exercise testing two weeks after infarct was found to be a safe procedure. We must emphasise, however, that we are concerned with patients who have had an uncomplicated myocardial infarction. The need for caution in interpreting our observations in all patients after infarction is obvious.

Correspondence should be addressed to: Dr G Jackson, Department of Cardiology, King's College Hospital, London SE5 9RS.

\section{References}

1 Theroux P, Water D, Halphen C, Debaisieux JC, Mizgala HF. Prognostic value of exercise testing soon after myocardial infarction. $N$ Engl.f Med $1979 ; 301: 341-5$

${ }^{2}$ Markiewicz W, Houston N, Debusk RF. Exercise testing soon after myocardial infarction. Circulation $1976 ; 56: 26-31$.

${ }^{3}$ Ellestad MH, Wan MKC. Predictive implications of stress testing: follow-up of 2700 subjects after maximum treadmill stress testing. Circulation $1975 ; 51: 363-9$.

${ }^{4}$ Castellanet MJ, Greenberg PS, Ellestad MH. Comparison of ST segment changes on exercise testing with angiographic findings in patients with prior myocardial infarction. Am F Cardiol 1978;42:29-35.

${ }^{5}$ Stiles G, Rosati R, Wallace A. Clinical relevance of exercise-induced ST segment elevation. Am 7 Cardiol 1980;46:931-6.

${ }^{6}$ European Coronary Surgery Study Group. Coronary artery bypass surgery in stable angina pectoris: survival at 2 years. Lancet 1979;ii: 839-42.

(Accepted 24 February 1982)
MEDICINES. - In an inflammation of the brain, nothing more certainly relieves the patient than a free difcharge of blood from the nofe. When this comes of its own accord, it is by no means to be ftopped, but rather promoted, by applying cloths dipped in warm water to the part. When bleeding at the nofe does not happen fpontaneoufly, it may be provoked, by putting a fraw, or any other fharp body up the noftril.

BLEEDING in the temporal arteries greatly relieves the head: but as this operation cannot always be performed, we would recommend in its ftead bleeding in the jugular veins. When the patient's pulfe and fpirits are fo low, that he cannot bear bleeding with the lancet, leeches may be applied to the temples. Thefe not only draw off the blood more gradually, but by being applied nearer to the part affected, generally give more immediate relief.

A DISCHARGE of blood from the hæmorrhoidal veins is likewife of great fervice, and ought by all means to be promoted. If the patient has been fubject to the bleeding piles, and that difcharge has been ftopped, every method muft be tried to reftore it; as the application of leeches to the parts, fitting over the fteams of warm water, Tharp clyfters or fuppofitories made of honey, aloes, and rock-falt.

If the inflammation of the brain be occafioned by the ftoppage of evacuations either natural or artificial, as the menfes, iffues, fetons, or fuch like, all means muft be ufed to reftore them as foon as poffible, or to fubftitute others in their ftead.

THE patient's body muft be kept open by ftimulating clyfters or fmart purges; and fmall quantities of nitre ought frequently to be mixed with his drink. Two or three drachms, or more, if the cafe be dangerous, may be ufed in the fpace of twenty-four hours.

THE head fhould be fhaved and frequently rubbed with vinegar and rofe-water. Cloths dipped in this mixture may likewife be applied to the temples. The feet ought frequently to be bathed in lukewarm water, and fort poultices of bread and milk may be kept conftantly applied to them.

If the difeafe proves obftinate, and does not yield to thefe medicines, it will be neceffary to apply a bliftering-plafter to the whole head.

(Buchan's Domestic Medicine, 1786.)

\section{Correction}

Improved control of brittle Parkinsonism by separate administration of levodopa and benserazide

We regret that some words were misplaced in this article by Drs McLellan and Dean (3 April, p 1001). The last sentence of the section "The patient" should have read: "A period of inpatient treatment with Madopar 62.5 (levodopa and benserazide hydrochloride) and bromocriptine did not improve her symptoms." 